\title{
On a certain class of abstract algebras
}

\author{
by \\ W. Narkiewicz (Wrockaw)
}

1. In [3], [5], [6], [7], [8] some classes of abstract algebras were investigated in which the notion of independence has the fundamental properties of linear independence. These classes rere: $v$-algebras (called also Marczewski's algebras) and $v^{*}$-algebras. For $v$-algebras a representation theorem was given by Urbanik [i]. A representation theorem for $v^{*}$-algebras having at least one independent triple of elements has also been given by Urbanik [8]. The general form of $v^{*}$-algebras without an independent triple is not known except in the case of algebras which have independent pairs of elements, are without an independent triple, have a set of fundamental operations consisting of operations of 2 raxiables and are without non-trivial operations of one variable. This case has been settled recently by Grätzer [2].

We shall say that a system $\left(a_{1}, \ldots, a_{n}\right)$ of elements of an algebra is $C$-independent if none of the $a_{i}$ 's belongs to the subalgebra generated by the others. (We assume the convention that the subalgebra generated by the void set consists of all algebraic constants, i.e. of values of constant algebraic operations. It follows that a single element is $C$-independent if and only if it is not an algebraic constant.)

The $v^{*}$-algebras can be defined as algebras in which the following two axioms hold:

(C) Independence in $\mathfrak{A}$ coincides with $C$-independence.

(Ex) If $b$ belongs to the subalgebra generated by $\left(a_{1}, \ldots, a_{n}\right)$, but does not belong to the subalgebra generated by $\left(a_{1}, \ldots, a_{n-1}\right)$, then $a_{n}$ belongs to the subalgebra generated by $\left(a_{1}, \ldots, a_{n-1}, b\right)$.

(Axiom (Ex) is the classical exchange axiom for linear independence.)

This definition does not coincide with the original definition given in [5] but their equivalence is easy provable.

In this paper we shall investigate, following a suggestion of Professor E. Marczewski, algebras in which only axiom (C) holds. These algebras we shall call $v^{* *}$-algebras. We obtain theorem II which is similar to theorem $\mathrm{I}$ in [5], which was obtained to characterize $v^{*}$-algebras. As a corollary wo infer that finite $v^{* *}$-algebras are $v^{*}$-algebras, i.e. that 
for finite algebras axiom ( $\mathbf{E x}$ ) is implied by (O). (Professor K. Urbar kindly communicated to me a direct proof of this statement. We reproduce his proof in $\$ 3$.) It should be remarked that the converse implication, i.e. $(\mathrm{Ex}) \rightarrow(\mathrm{C})$ for finite algebras, is not true. Indeed, let $X=(a, b, c)$ and let the only fundamental operation be $f(x, y)$ defined by equations $f(a, b)=f(b, a)=a, f(a, c)=f(c, a)=c, f(b, c)=f(c, b)=b, f(x, x)=x$. In the algebra thus defined every pair of elements is dependent, consequently (Ex) holds but (C) does not hold, as every subset of $X$ is a subalgebra.

We also give a full description of $v^{* *}$-algebras having only operations depending on at most one variable.

For terminology and notation used here, see [4] and [5]. In particular $\boldsymbol{A}^{(n)}$ is the set of all algebraic operations of $n$ variables of the algebra $\mathfrak{A}=(A, A)$ and $\left[a_{1}, \ldots, a_{n}\right]$ is the subalgebra of $\mathfrak{A}$ generated by the set $\left(a_{1}, \ldots, a_{n}\right)$. We shall also write $\left(a_{1}, \ldots, \hat{a}_{j}, \ldots, a_{n}\right)$ instead of $\left(a_{1}, \ldots, a_{j-1}\right.$, $\left(a_{j \div 1}, \ldots, a_{n}\right)$ and instead of $e_{k}^{(n)}\left(x_{1}, \ldots, x_{n}\right)$ we shall often write simply $x_{k}$.

2. Now we shall give some examples of $v^{* *-a l g e b r a s ~ w h i c h ~ a r e ~}$ not $v^{*}$-algebras.

(a) Let $R$ be an associative ring with the unit element, withont divisors of zero, satisfying the following condition:

For every pair $a, b$ from $R$ there exists a $e$ in $R$ such that $a=a b$ of $b=c a$.

Let $L$ be a left-module over $R, I I$ - the set of all elementis of $C$ which are left-divisible by each non-zero element of $R$, and finally let $I_{0}$ be an arbitrary submodule of $M$.

If $\boldsymbol{A}$ is the class of operations $f$ defined by

$$
f\left(x_{1}, \ldots, x_{n}\right)=\sum_{k=1}^{n} \lambda_{k} x_{k}+a \quad\left(\lambda_{1}, \ldots, \lambda_{n} \in R, a \in \mathbb{M} M_{0}\right),
$$

then $(L, \boldsymbol{A})$ is a $v^{* *}$-algebra.

If $\boldsymbol{A}$ is the class of operations $f$ defined by

$$
f\left(x_{1}, \ldots, x_{n}\right)=\sum_{k=1}^{n} \lambda_{k} x_{k}+a \quad\left(\lambda_{j} \in R, \sum_{k=1}^{n} \lambda_{k}=1, a \in M I_{0}\right),
$$

then $(L, \boldsymbol{A})$ is a $v^{* *}$-algebra.

Evidently, if $R$ is not a division ring, the algebras obtained in, this way cannot be $v^{*}$-algebras.

(b) Let $X$ be any non-empty set, and $S$ a semigroup of transformations of $X$ satisfying the following conditions:

(i) The identical transformation belongs to $S$

(ii) The left-cancellation law holds in $S$.

(iii) For any four elements $f, g, F, G$ of $S$ from $f g=F G$ it follows that, with a suitable $H$ from $S, g=H G$ or $G=H g$.
Let $X_{0}=\left\{a \in X: \bigvee_{s_{1} \neq s_{2} \in S} s_{1}(a)=s_{2}(a)\right\}$ and let $Y$ be a subset of $X$ containing $X_{0}$ and such that $S(Y) \subset Y$. Now if $\boldsymbol{A}$ is the class of all operations $f$ defined by

$$
\begin{aligned}
& f\left(x_{1}, \ldots, x_{n}\right)=s\left(x_{j}\right) \quad(1 \leqslant j \leqslant n, s \in \mathbb{S}) \\
& f\left(x_{1}, \ldots, x_{n}\right)=a \quad(a \in Y)
\end{aligned}
$$

then $(X, \boldsymbol{A})$ is a $v^{* *}$-algebra. If $S$ is not a group, then that algebra cannot be a $v^{*}$-algebra. We shall prove later that almost every $v^{* *}$-algebra in which all operations depend on at most one variable can be constructed in such a way.

From theorem III proved below and from the representation theorem for $v^{*}$-algebras containing at least one independent triplet, proved by Urbanik [8], it follows that a finite $v^{* *}$-algebra containing at least one independent triplet of elements is of the form (a) or (b). The problem can be posed whether every $v^{* *}$-algebra containing at least one independent triplet is of the form (a) or (b). We have not been able to prove this or to give a counter-example.

3. Let $\mathfrak{A}$ be a $v^{* *-a l g e b r a . ~ T h e ~ f o l l o w i n g ~ t h r e e ~ s t a t e m e n t s ~ a r e ~ e a s y ~}$ consequences of $(\mathbf{C})$ :

(i) Every subalgebra of $\mathfrak{A}$ is also a $v^{* *}$-algebra.

(ii) If $\mathfrak{A}$ has an independent $n$-tuple of elements, then the algebra $\boldsymbol{A}^{(n)}$ is also a $v^{* * \text {-algebra. }}$

(iii) Every selfdependent element in $\mathfrak{A}$ is an algebraic constant. Now we prove

THEOrEn I. If a $v^{* *-a l g e b r a ~ h a s ~ a ~ b a s i s, ~ t h e n ~ a l l ~ b a s e s ~ h a v e ~ t h e ~ s a m e ~}$ number of elements.

Proof. If $\mathfrak{A}$ has an infinite basis, then all bases have the same cardinal number (see [4], p. 50). Suppose thus that $\mathfrak{A}$ has two finite bases with a different number of elements, say $\left(a_{1}, \ldots, a_{k}\right)$ and $\left(b_{1}, \ldots, b_{m}\right)$. Let $m>k$. Then there exist algebraic operations $f_{1}\left(x_{1}, \ldots, x_{k}\right), \ldots, f_{m}\left(x_{1}, \ldots, x_{k}\right)$, $g_{1}\left(x_{1}, \ldots, x_{m}\right), \ldots, g_{k}\left(x_{1}, \ldots, x_{m}\right)$ such that $f_{i}\left(a_{1}, \ldots, a_{k}\right)=b_{i}(i=1,2, \ldots, m)$ and $g_{j}\left(b_{1}, \ldots, b_{m}\right)=a_{j}(j=1,2, \ldots, k)$; consequently

$$
f_{i}\left(g_{1}\left(x_{1}, \ldots, x_{m}\right), \ldots, g_{k}\left(x_{1}, \ldots, x_{m}\right)\right)=x_{i} \quad(i=1,2, \ldots, m),
$$

and so the set $\left(g_{1}, \ldots, g_{k}\right)$ is a set of generators of the algebra $\boldsymbol{A}^{(m)}$. Let us now define

$$
h_{y}\left(x_{1}, \ldots, x_{m-1}\right)=y_{j}\left(x_{1}, \ldots, x_{m-1}, x_{m-1}\right) \quad(j=1,2, \ldots, k) .
$$

Then evidently

$$
f_{m}\left(h_{1}, \ldots, h_{k}\right)=x_{m-1}=f_{m-1}\left(h_{1}, \ldots, h_{k}\right),
$$


whence (as $f_{n} \neq f_{m-1}$ ) the system $\left(h_{1}, \ldots, h_{k}\right)$ is dependent in $\boldsymbol{A}^{(m-1)}$ From (ii) we infer that $\boldsymbol{A}^{(m-1)}$ is a $v^{* *}$ - algebra; consequently with a suitable $F \in A^{(k-1)}$ and some $j$ we have

$h_{j}\left(x_{1}, \ldots, x_{m-1}\right)=F\left(h_{1}\left(x_{1}, \ldots, x_{m-1}\right), \ldots, \hat{h}_{j}\left(x_{1}, \ldots, x_{m-1}\right), \ldots, h_{k}\left(x_{1}, \ldots, x_{m-1}\right)\right)$.

As $\left[h_{1}, \ldots, h_{k}\right]=\boldsymbol{A}^{(m-1)}$, we have $\left[h_{1}, \ldots, \hat{h}_{f}, \ldots, h_{k}\right]=\boldsymbol{A}^{(m-1)}$. If the system $\left(h_{1}, \ldots, \hat{h}_{j}, \ldots, h_{k}\right)$ is dependent, we can remove some elements from it and obtain a basis $\left(h_{i_{1}}, \ldots, h_{i_{t}}\right)$ of $\boldsymbol{A}^{(m-1)}$. (We obtain a non-void basis, in any case, as $\boldsymbol{A}^{(m-1)}$ cannot be generated by an operation which is selfdependent, i.e. constant (by (iii)).) So far we have proved that, if a $v^{* *}$-algebra $\mathfrak{A}$ has bases of $k$ resp. $m$ elements, then $\boldsymbol{A}^{(m-1)}$ las two bases consisting of $t \leqslant k-1$ of course) and $m-1$ elements, respectively. By repeating this argument we finally obtain a $v^{* *}$-algebra $\boldsymbol{A}^{(r)}$ with two bases consisting of $r \neq 1$ and 1 elements, respectively. We can now apply theorem 8 of [2] to find that $\boldsymbol{A}^{(r)}$ has a system of selfdependent generators. But it is incompatible with (iii) as $\boldsymbol{A}^{(r)}$ would then consist of constant operations only. The contradiction obtained proves the theorem.

It should be remarked that there exist $v^{* *}$-algebras withont any basis. For example, let $\mathfrak{A}=(X, F)$ where $X$ is the set of rational integers and $\boldsymbol{F}$ consists of all functions $f(x)=x+a$, where $a$ is a positive rational integer. However, perhaps every $v^{* *}$-algebra having operations of 2 variables which depend on every rariable has a basis.

4. Let $\mathfrak{U}=(A, \boldsymbol{A})$ be any algebra. By $\Delta_{k}$ we shall denote the set of ordered $k$-tuples of algebraic operations of $k$ variables which are $O$-independent in the algebra $\boldsymbol{A}^{(k)}$. Similarly, by $\nabla_{k}$ we shall denote the set of ordered $k$-tuples of elements of $\mathfrak{A}$ which are $C$-independent. (It should be remarked that in view of the convention assumed in $\S 1$ the definitions of $\Delta_{k}$ and $\nabla_{k}$ are formally different for $k=1$ and $k \neq 1$.) In [5] the following theorem was proved:

The algebra $\mathfrak{U}$ is a $v^{*}$-algebra if and only if $\Delta_{k}$ is a group of transformations $\nabla_{k} \rightarrow \nabla_{k}$ whenever $\nabla_{k}$ is non-roid and, moreover, $A_{1}$ has no fixed points.

(The set $\Delta_{k}$ is here treated as a set of transformations acting from $\nabla_{k}$ into the set of all $k$-tuples of elements of the algebra as follows: $\left.\left(f_{1}, \ldots, f_{k}\right)\left(\left(a_{1}, \ldots, a_{k}\right)\right)=\left(f_{1}\left(a_{1}, \ldots, a_{k}\right), \ldots, f_{k}\left(a_{1}, \ldots, a_{k}\right)\right).\right)$

We now prove a similar result for $v^{* *}$-algebras, namely:

THEOREM II. If the algebra $\mathfrak{A}$ is a $v^{* *}$-algebra, then:

(*) $\Delta_{k}$ is a semigroup of transformations $\nabla_{k} \rightarrow \nabla_{k}$ whenever $\nabla_{k}$ is nonvoid and, moreover, in $\Delta_{1} f(a)=g(a)$ implies $f=g$.

Conversely, if an algebra $\mathfrak{A}$ satisfies condition (*), then $\mathfrak{A}$ is a $v^{* *}$-algebra.

Proof. The first part of this theorem follows immediately from the lact that in a $v^{* *}$ - algebra the set $\Delta_{k}$ is exactly the set of all independent ordered $k$-tuples of operations from $\boldsymbol{A}^{(k)}$ and $\nabla_{k}$ is the set of all independent ordered $k$-tuples of elements.

Suppose now that the algebra $\mathfrak{A}=(\boldsymbol{A}, \boldsymbol{A})$ satisfies (*). For convenience we break the proof into several lemmas.

Lenna 1. Every selfdependent element of $\mathfrak{A}$ is an algebraic constant.

Let $f(x) \neq g(x), f(a)=g(a)=b$, and $a \in \nabla_{1}$. In view of $(*) f, g \in \Delta_{1}$ is impossible. If $f, g \notin \Delta_{1}$, then $f(x)=b=g(x)$. If $f \in \Delta_{1}, g \notin \Delta_{1}$, then $g(x)=b$; thus $b \notin \nabla_{1}$, but $b=f(a) \in \nabla_{1}$ by $(*)$, which is a contradiction.

Lenard 2. Suppose that $\left(a_{1}, \ldots, a_{n}\right) \in \Delta_{n}$ and that, for every $k(1 \leqslant k \leqslant n)$ s the system $\left(a_{1}, \ldots, \hat{a}_{k}, \ldots, a_{n}\right)$ is independent. If for some $f, g \in \boldsymbol{A}^{(n)}, f\left(a_{1}, \ldots, a_{n}\right)$ $=g\left(a_{1}, \ldots, a_{n l}\right)=b$, and $f\left(x_{1}, \ldots, x_{n}\right) \neq g\left(x_{1}, \ldots, x_{n}\right)$, then $b$ is not an algebraic constant.

Suppose that $b$ is an algebraic constant and that $f\left(x_{1}, \ldots, x_{n}\right)$ is not a constant operation. Then $f$ has to depend on $x_{1}$, for otherwise the system $\left(a_{2}, \ldots, a_{n}\right)$ would be dependent. Let $F=\left(f, x_{2}, \ldots, x_{n}\right)$. If $F \in \Delta_{n}$ then $\left(b, a_{2}, \ldots, a_{n}\right)=F\left(\left(a_{1}, \ldots, a_{n}\right)\right) \in \nabla_{n}$, which is impossible since $b \in\left[a_{2}, \ldots, a_{n}\right]$. Hence $F \notin \Delta_{n}$ and, since $f$ depends on $x_{1}$, with suitable $j$ and $H \in A^{(n-1)}$, we have $x_{j}=\boldsymbol{H}\left(f, x_{2}, \ldots, x_{j}, \ldots, x_{n}\right)$. By putting in the last equation $x_{i}=a_{i}$ for $i=1,2, \ldots, n$ we obtain a contradiction of $\left(a_{1}, \ldots, a_{n}\right) \in \nabla_{n}$.

Our theorem will be proved if we show that $\left(a_{1}, \ldots, a_{n}\right) \in \nabla_{n}$ implies the independence of the system $\left(a_{1}, \ldots, a_{n}\right)$. We shall prove this by induction. For $n=1$ this statement is true by lemma 1 . The following lemma proves this statement for $n=2$ (this must be done in view of the remark after the definition of $\Delta_{n}$ and $\nabla_{n}$ ).

Lenna 3. If the system $\left(a_{1}, a_{2}\right)$ is dependent, then it is also $O$-dependent.

Suppose that $f\left(a_{1}, a_{2}\right)=g\left(a_{1}, a_{2}\right)=b, f\left(x_{1}, x_{2}\right) \neq g\left(x_{1}, x_{2}\right)$ and that the pair $\left(a_{1}, a_{2}\right)$ is $C$-independent, i.e. belongs to $\nabla_{2}$. Then $(f, g)$ does not belong to $\Delta_{2}$ (otherwise $\left.(b ; b)=(f, g)\left(\left(a_{1}, a_{2}\right)\right) \epsilon \nabla_{2}\right)$; consequently with some $G(x) \in A^{(1)}, f\left(x_{1}, x_{2}\right)=G\left(g\left(x_{1}, x_{2}\right)\right)$ or $g\left(x_{1}, x_{2}\right)=G\left(f\left(x_{1}, x_{2}\right)\right)$. In both cases by putting $x_{1}=a_{i}(i=1,2)$ one obtains $b=G(b)$, and since $G(x) \neq x$, we find that $b$ is selfdependent, and from lemma 1 it follows that $b$ must be an algebraic constant, contrary to lemma 2 .

Now comes the inductional step. Tet us assume that for all $m<n$ we have already proved the following:

(\&) If the $m$-tuple $\left(a_{1}, \ldots, a_{m}\right)$ is dependent, then it is also $C$-dependent.

Let $f\left(a_{1}, \ldots, a_{n}\right)=g\left(a_{1}, \ldots, a_{n}\right)=b, f \neq g$, and suppose that the system $\left(a_{1}, \ldots, a_{n}\right)$ is $C$-independent, i.e. belongs to $\nabla_{n}$. We can assume that, for every $k \leqslant n$, the system $\left(a_{1}, \ldots, \hat{a}_{k}, \ldots, a_{n}\right)$ is independent. 
Lennca 4. For every $(n-2)$-tuple of indices $1 \leqslant i_{1}, \ldots, i_{n-2} \leqslant n$ one of the following equations holds:

(A) $b=\varphi\left(a_{i_{1}}, \ldots, a_{i_{n-2}}\right)$ with suitable $\varphi \in \boldsymbol{A}^{(n-2)}$

(B) $a_{i_{j}}=\psi\left(b, a_{i_{1}}, \ldots, \hat{a}_{i j}, \ldots, a_{i_{n-2}}\right)$ with some $j(1 \leqslant j \leqslant n-2)$ and suitable $\psi \in \boldsymbol{A}^{(n-2)}$.

Evidently $\left(f, g, x_{i_{1}}, \ldots, x_{i_{n-2}}\right)$ does not belong to $A_{n}$; consequently there exists a $\Phi \in A^{(n-1)}$ such that one of the following three equations holds:

(i) $f\left(x_{1}, \ldots, x_{n}\right)=\Phi\left(g\left(x_{1}, \ldots, x_{n}\right), x_{i_{1}}, \ldots, x_{i_{n-2}}\right)$,

(ii) $g\left(x_{1}, \ldots, x_{n}\right)=\Phi\left(f\left(x_{1}, \ldots, x_{n}\right), x_{i_{1}}, \ldots, x_{i_{1-2}}\right)$,

(iii) $\quad x_{i j}=\Phi\left(f\left(x_{1}, \ldots, x_{n}\right), g\left(x_{1}, \ldots, x_{n}\right), x_{i_{1}}, \ldots, \hat{x}_{i j}, \ldots, x_{i_{n-2}}\right)$ with some $j$.

If (iii) then (B) holds with $\psi\left(x_{1}, \ldots, x_{n-1}\right)=\Phi\left(x_{1}, x_{1}, x_{2}, \ldots, x_{n-1}\right)$. In the other cases we see that the system $\left(b, a_{i_{1}}, \ldots, a_{i_{n-2}}\right)$ is dependent, for otherwise, by substitution $x_{i}=a_{i}$, we should have $\Phi\left(x_{1}, \ldots, x_{n}\right)=x_{1}$, which means that $f=g$, contrary to our assumption. The dependence of $\left(b, a_{i_{1}}, \ldots, a_{i_{n-2}}\right)$ by the inductional assumption implies the truth of one of the equation (A), (B).

Lemma 5. If $F^{\prime}\left(x_{1}, \ldots, x_{n}\right) \in \boldsymbol{A}^{(n)}$; and $F \neq e_{j}^{(n)}$ then $F^{\prime}\left(a_{1}, \ldots, a_{n}\right) \neq a_{j}$ for $j=1,2, \ldots, n$ and $n=2,3, \ldots$

Suppose that $F\left(a_{1}, \ldots, a_{n}\right)=a_{j}$. Let $k \neq j$. Then the $n$-timple $\left(F, x_{1}, \ldots, \hat{x}_{k}, \ldots, x_{n}\right)$ does not belong to $\Delta_{n}$. If the operation $F^{r}$ does not depend on $x_{k}$, then the system $\left(a_{1}, \ldots, \hat{a}_{k}, \ldots, a_{n}\right)$ is dependent, but this is excluded by assumption. Thus $F$ depends on $x_{k}$ and it exists $r(1 \leqslant r \leqslant n)$ such that with a suitable $G \in \boldsymbol{A}^{(n-1)}$

$$
x_{r}=G\left(F^{\prime}\left(x_{1}, \ldots, x_{n}\right), x_{1}, \ldots, \hat{x}_{k}, \ldots, \hat{x}_{r}, \ldots, x_{n}\right),
$$

whence $a_{r}=G\left(a_{j}, a_{1}, \ldots, \hat{a}_{k}, \ldots, \hat{a}_{r}, \ldots, a_{n}\right)$

The last equation can occur only if $j=r$ and $G\left(x_{1}, \ldots, x_{n-1}\right)=x_{1}$, but then $F\left(x_{1}, \ldots, x_{n}\right)=x_{r}$; consequently $F^{\prime}$ does not depend on $x_{k}$. The contradiction obtained proves the lemma.

LEMra 6. For every pair of indices $k, r(1 \leqslant k \neq r \leqslant n)$ there exists an operation $H \in A^{(n)}$ such that $H\left(a_{1}, \ldots, a_{n}\right)=b$ and

$$
A_{I I}=\left[H, e_{1}^{(n)}, \ldots, \hat{e}_{k}^{(n)}, \ldots, \hat{e}_{r}^{(n)}, \ldots, e_{n}^{(n)}\right] \neq \boldsymbol{A}^{(n)} .
$$

Suppose that for a pair $k, r$ the lemma is not true. Then every operation $Z \in A^{(n)}$ such that $Z\left(a_{1}, \ldots, a_{n}\right)=b$ must depend on $x_{k}$ and $x_{r}$, for otherwise $e_{k}^{(n)}$ or $e_{r}^{(n)}$ cannot belong to $A_{Z}$. Now we prove the following statement:
(\#) If there exists a $k$-tuple of different indices $j_{1}, \ldots, j_{k}\left(1 \leqslant j_{\alpha} \leqslant n\right)$ such that for every operation, $Z \in A^{(n)}$ satisfying $Z\left(a_{1}, \ldots, a_{n}\right)=b$

$$
\left[Z, e_{1}^{(n)}, \ldots, \hat{e}_{j_{1}}^{(n)}, \ldots, \hat{e}_{j_{2}}^{(n)}, \ldots, \hat{e}_{j_{k}}^{(n)}, \ldots, e_{n}^{(n)}\right]=\boldsymbol{A}^{(n)}
$$

then one can choose from the remaining indices $\leqslant n$ such an index $j_{k+1}$ that for every operation $Z \in A^{(n)}$ satisfying $Z\left(a_{1}, \ldots, a_{n}\right)=b$ :

$$
\left[Z, e_{1}^{(n)}, \ldots, \hat{e}_{j_{1}}^{(n)}, \ldots, \hat{e}_{j_{2}}^{(n)}, \ldots, \hat{e}_{j_{k}}^{(n)}, \ldots, \hat{e}_{j_{k+1}}^{(n)}, \ldots, e_{n}^{(n)}\right]=A^{(n)} .
$$

Let $S\left(a_{1}, \ldots, a_{n}\right)=I^{\prime}\left(a_{1}, \ldots, a_{n}\right)=b$ and $S \neq T$. Then with a suitable $F^{H} \in A^{(n-k+1)}$ we have

$$
S\left(x_{1}, \ldots, x_{n}\right)=W\left(T\left(x_{1}, \ldots, x_{n}\right), x_{1}, \ldots, \hat{x}_{j_{1}}, \ldots, \hat{x}_{j_{k}}, \ldots, x_{n}\right) .
$$

By putting here $x_{i}=a_{i}(i=1, \ldots, n)$ we obtain

$$
b=W^{\prime}\left(b, a_{1}, \ldots, \hat{a}_{j_{1}}, \ldots, \hat{a}_{j_{k}}, \ldots, a_{n}\right),
$$

and so the system $\left(b, a_{1}, \ldots, \hat{a}_{j_{1}}, \ldots, \hat{a}_{j_{k}}, \ldots, a_{n}\right)$ is dependent (because $F=e_{1}^{(n)}$ would imply $S=T^{\prime}$ ). From the inductional assumption follows the truth of one of the following two equations:

(a) $b=G\left(a_{1}, \ldots, \hat{a}_{j_{1}}, \ldots, \hat{a}_{j_{k}}, \ldots, a_{n}\right)$ with a suitable $G \in \boldsymbol{A}^{(n-k)}$,

(b) $a_{s}=R\left(b, a_{1}, \ldots, \hat{a}_{j_{1}}, \ldots, \hat{a}_{j_{k}}, \ldots, \hat{a}_{s}, \ldots, a_{n}\right)$ with a suitable $s$ and $R \in A^{(n-k)}$.

Case (a) is excluded by the remark at the beginning of this lemma. In case (b) we infer by the use of lemma 5 that

$$
x_{s}=R\left(T\left(x_{1}, \ldots, x_{n}\right), x_{1}, \ldots, \hat{x}_{j_{1}}, \ldots, \hat{x}_{j_{k}}, \ldots, \hat{x}_{s}, \ldots, x_{n}\right) ;
$$

consequentily

$$
\left[T, e_{1}^{(n)}, \ldots, \hat{e}_{j_{1}}^{(n)}, \ldots, \hat{e}_{l_{i}}^{(n)}, \ldots, \hat{e}_{s}^{(n)}, \ldots, e_{n}^{(n)}\right]=\boldsymbol{A}^{(n)}
$$

From the arbitrariness of $T\left(x_{1}, \ldots, x_{n}\right)$ the statement (\#) follows by putting $j_{k+1}=8$. As the assumption of $(\#)$ is satisfied for $k=2$, we infer by induction that for each operation $Z\left(x_{1}, \ldots, x_{n}\right) \in \boldsymbol{A}^{(n)}$ such that In must have $[Z]=A^{(n)}$. In particular, with some $K(x) \in \dot{A}^{(1)}$ we have $f\left(x_{1}, \ldots, x_{n}\right)=K\left(g\left(x_{1}, \ldots, x_{n}\right)\right)$ and so $K(b)=b$ By lemma $1, b$ is an algebraic constant, which contradicts lemma 2 . This ends the proof of lemma 6.

Now we can finish the proof of theorem II. If for no set of indices an equation of the form (B) holds, then in view of lemma 4 for each $(n-2)$-tuple of indices equations (A) hold and so:

$$
b=p_{i j}\left(a_{1}, \ldots, \hat{a}_{i}, \ldots, \hat{a}_{j}, \ldots, a_{n}\right)
$$

for each pair $i \neq j$. From the independence of every system $\left(a_{1}, \ldots, \hat{a}_{i}, \ldots, a_{n}\right)$ fols an algebraic constant, contrary to lemma 2 . Thus 
for a certain $(n-2)$-tuple of indices an equation of the form (B) holds:

(1)

$$
a_{k}=h_{1}\left(b, a_{i_{1}}, \ldots, a_{i_{n-3}}\right) \quad\left(i_{j} \neq k, j=1, \ldots, n-3\right) .
$$

Consider the $(n-2)$-tuple $\left(a_{j_{1}}, \ldots, a_{j_{n-3}}\right)$ where each $j_{l}$ is different, from $k$. If it were $H\left(a_{j_{1}}, \ldots, a_{j_{n-2}}\right)=b$ with some $H \in A^{(n-2)}$, then by substituting the last equation into (1) we would obtain a contradiction. with the $C$-independence of $\left(a_{1}, \ldots, a_{n}\right)$. Thus we must have

$$
a_{j_{t}}=h_{2}\left(b, a_{j_{1}}, \ldots, \hat{a}_{j_{t}}, \ldots, a_{j_{n-3}}\right)
$$

with some $t$ and $h \in \boldsymbol{A}^{(n-2)}$.

Let $r$ be the only number not greater than $n$ which does not ocem in the sequence $j_{1}, \ldots, j_{n-2}, k$ and let us write

$$
R_{T}=\left[T, e_{j_{1}}^{(n)}, \ldots, \hat{e}_{j_{t}}^{(n)}, \ldots, e_{j_{n-3}}^{(n)}, e_{r}^{(n)}\right]
$$

for every $T$ such that $T\left(a_{1}, \ldots, a_{n}\right)=b$. From (2) and lemma 5 we infer that $e_{j_{t}}^{(n)} \in R_{T}$ and, moreover, by (1) and lemma 5 we have $e_{i}^{(n)} \in R_{T}$, whence $R_{T}=A^{(n)}$ for every operation $T$ such that $T\left(a_{1}, \ldots, a_{n}\right)=b$. But this contradicts lemma 6 . The contradiction obtained proves om theorem.

Remark. In the semigroups $\Delta_{n}$ the left-cancellation law holds. Indeed, let $g f=h f$, where $f=\left(f_{1}, \ldots, f_{n}\right), y=\left(g_{1}, \ldots, g_{n}\right), h=\left(h_{1}, \ldots, h_{n}\right)$. Then $y_{j}\left(f_{1}\left(x_{1}, \ldots, x_{n}\right), \ldots, f_{n}\left(x_{1}, \ldots, x_{n}\right)\right)=h_{w}\left(f_{1}\left(x_{1}, \ldots, x_{n}\right), \ldots, f_{n}\left(x_{1}, \ldots, x_{n}\right)\right)$ for each $j=1, \ldots, n$. As the system $\left(f_{1}, \ldots, f_{n}\right)$ is independent, thus $g_{j}=h_{i j}$ for each $j$, i.e. $f=g$. As a corollary (we use here only the trivial "only if" part of theorem II) follows

THEorem III. A finite $v^{* *}$-algebra is a $v^{*}$-algebra.

For the proof it suffices to remark that a transformation semigroup of a finite set containing the identical transformation and with the left cancellation law is a group.

The following direct proof of this theorem is due to Professor $\mathrm{K}$. $\mathrm{Ur}$ banik. Let $\mathfrak{X}=\left[c_{1}, \ldots, c_{n}\right]$. Then every independent $n$-tuple is a basis for $\mathfrak{A}$ because from $\left[b_{1}, \ldots, b_{n}\right] \subset \mathfrak{A}$ and from independence of the set $\left(b_{1}, \ldots, b_{n}\right)$ follows $\left[b_{1}, \ldots, b_{n}\right] \approx \boldsymbol{A}^{(n)} \approx \mathfrak{A}$, whence, from the finiteness of $\mathfrak{A}$ $\left[b_{1}, \ldots, b_{n}\right]=\mathfrak{A}$. The same is true for every subalgebra of $\mathfrak{A}$. Now let $\left(a_{1}, \ldots, a_{m}\right)$ be an independent set, and $\left(a_{1}, \ldots, a_{m}, a_{m+1}\right)$ a dependent set. Then, for some $i, a_{i} \in\left[a_{1}, \ldots, \hat{a}_{i}, \ldots, a_{m+1}\right]$, whence $B_{1}=\left[a_{1}, \ldots, a_{m}\right]$ $\subset\left[a_{1}, \ldots, \hat{a}_{i}, \ldots, a_{m+1}\right]=B_{2}$. Therefore $B_{2}$ has a basis of $r=m$ elements and so $\left[a_{1}, \ldots, a_{r}\right]=B_{2}$; comsequently $a_{m+1} \in B_{1}$, q.e.d.

THEOREM IV. If $\mathfrak{A}$ is a $v^{* *}$-algebra in which every operation, depends. on at most one variable, then $\mathfrak{A}$ has the form given in $\$ 2,(\mathrm{~b})$, or each element of the algebra is an algebraic constant.
It suffices to prove that condition (iii) $(\$ 2,(b))$ holds. Suppose that $\nabla_{2}$ is non-roid and that $f(x), g(x), F(x), G(x)$ are non-constant algebraic operations such that $f(g(x))=F^{\prime}(G(x))$. Suppose, moreover, that the pair $(g(x), G(x))$ belongs to $\Delta_{2}$. Then, since evidently $(f(x), F(y)) \epsilon \Delta_{2}$, we have $(f(g(x)), f(g(x)))=\left(f(x), F^{\prime}(y)\right) \cdot(g(x), G(x)) \in \Delta_{2}$, which is clearly impossible. Now let $\nabla_{2}$ be void and let $a$ be an element which is not an algebraic constant. Let $f(x), g(x)$ be arbitrary non-constant operations of one variable. Since $\nabla_{2}$ is void, with some non-constant $h(x)$ we must have $f(a)=h(g(a))$ or $g(a)=h(f(a))$. From the independence of the system (a) it follows that, for every $x$ in $A, f(x)=h(g(x))$ or $g(x)=h(f(x))$, which ends the proof.

4. In this part we shall show that some conditions imposed on a $v^{* *}$-algebra imply that it must be a $v^{*}$-algebra.

(i) If $\mathfrak{Y}$ is a $v^{* *}$-algebra in which for some $n$ (finite or not) every independent n-tuple is a basis, then $\mathfrak{A}$ is a $v^{*}$-algebra.

Proof. If $n$ is an infinite cardinal number, then $\left(a_{1}, a_{2}, \ldots\right)$ and $\left(a_{2}, a_{3}, \ldots\right)$ have to be bases, which is clearly impossible. Now let $n$ be finite. It suffices to prove that $\Delta_{k}$ is a group for every $k \leqslant n$.

Lema. If $\Delta_{k}$ is a group, then $\Delta_{k-1}$ is also a group.

We can imbed $\Delta_{k-1}$ isomorphically in $\Delta_{k}$ as follows:

$$
\begin{aligned}
i:\left(f_{1}\left(x_{1}, \ldots, x_{n-1}\right), \ldots, f_{n-1}\left(x_{1}, \ldots, x_{n-1}\right)\right) \rightarrow & \\
& \rightarrow\left(f_{1}\left(x_{1}, \ldots, x_{n-1}\right), \ldots, f_{n-1}\left(x_{1}, \ldots, x_{n-1}\right), x_{n}\right) .
\end{aligned}
$$

Let $Z=i\left(\Delta_{Z-1}\right)$. It suffices to prove that $\alpha \in Z$ implies $\alpha^{-1} \in Z$. Let $\alpha=\left(f_{1}, \ldots, f_{n-1}, x_{n}\right)$ and $a^{-1}=\left(g_{1}, \ldots, g_{n}\right)$. (Here $f_{j}, g_{j}$ belong to $A^{(n)}$, but the $f_{j}$ 's depend only on the first $n-1$ variables.) Then

$$
g_{j}\left(f_{1}\left(x_{1}, \ldots, x_{n-1}\right), \ldots, f_{n-1}\left(x_{1}, \ldots, x_{n-1}\right), x_{n}\right)=x_{j} \quad(j=1,2, \ldots, n),
$$

(3) $f_{j}\left(g_{1}\left(x_{1}, \ldots, x_{n}\right), \ldots, g_{n}\left(x_{1}, \ldots, x_{n}\right)\right)=x_{j} \quad(j=1,2, \ldots, n-1)$,

$g_{n}\left(x_{1}, \ldots, x_{n}\right)=x_{\eta_{n}}$

Let $g_{j}^{\prime}\left(x_{1}, \ldots, x_{n-1}\right)=g_{j}\left(x_{1}, \ldots, x_{n-1}, x_{n-1}\right)$ for $j=1,2, \ldots, n-1$. Then evidently

(4) $f_{j}\left(g_{1}^{\prime}\left(x_{1}, \ldots, x_{n-1}\right), \ldots, g_{n-1}^{\prime}\left(x_{1}, \ldots, x_{n-1}\right)\right)=x_{j} \quad(j=1,2, \ldots, n-1)$.

By putting, in (3), $x_{j}=g_{j}^{\prime}\left(x_{1}, \ldots, x_{n-1}\right)$ for $j=1,2, \ldots, n-1$ we. obtain, by the use of $(4), g_{j}\left(x_{1}, \ldots, x_{n-1}, x_{n}\right)=g_{j}^{\prime}\left(x_{1}, \ldots, x_{n-1}\right)$, and so $a^{-1}=\left(g_{1}^{\prime}, \ldots, g_{n-1}^{\prime}, x_{n}\right) \in Z$.

In view of this lemma it suffices to prove that $\Delta_{n}$ is a group. Let $f \in \Delta_{n}, f=\left(f_{1}, \ldots, f_{n}\right)$, and let $\left(a_{1}, \ldots, a_{n}\right) \in \nabla_{n}$. Let $b_{j}=f_{j}\left(a_{1}, \ldots, a_{n}\right)$, $j=1, \ldots, n$. The system $\left(b_{1}, \ldots, b_{n}\right)$ is independent, and so it is a basis; 
consequently with suitable $g_{1}, \ldots, g_{n}$ we have $a_{j}=g_{j}\left(b_{1}, \ldots, b_{n}\right), j=1, \ldots, n$. Since the system $\left(g_{1}, \ldots, g_{n}\right)$ is evidently independent, it follows from the independence of the systems $\left(a_{1}, \ldots, a_{n}\right)$ and $\left(b_{1}, \ldots, b_{n}\right)$ that $\left(y_{1}, \ldots, y_{n}\right)$ $=f^{-1}$, which ends the proof.

(ii) If $\mathfrak{A}$ is a $v^{* *-a l g e b r a ~ w i t h ~ a ~ f i n i t e ~ b a s i s ~ i n ~ w h i c h ~ e v e r y ~ i n d e p e n d e n t ~}$ set can be extended to a basis, then $\mathfrak{A}$ is a v*-algebra.

Pr oof. Let $\mathfrak{A}$ possess a basis of $n$ elements. Then every independent $n$-tuple forms a basis (because it can be extended to a basis and from theorem I it follows that every basis has $n$ elements) and it remains to apply the foregoing statement.

(iii) If $A$ is a $v^{* *}$-algebra with a finite basis and $\mathfrak{A}$ does not contain a subalgebra different from $\mathfrak{A}$ but with $\mathfrak{A}$ isomor'phie, then $\mathfrak{A}$ is a $v^{*}$-algebra.

This statement is an immediate consequence of (i), since it follows from the assumption that every independent $n$-tuple (where $n$ is the power of the basis) is a basis.

The last statement shows that if we introduce the notion of dimension for $v^{* *}$-algebra with a finite basis as the cardinal number of the basis (which is well-defined in view of theorem I), then the dimension of a subalgebra can be equal to the dimension of the algebra and that this peculiarity does not occur only for $v^{*}$-algebras.

Statement (iii) is not true for algebras with an infinite basis, becurtse $\left[a_{1}, a_{2}, \ldots\right] \approx\left[a_{2}, \ldots\right]$. We do not know whether (ii) is false for algebras with an infinite basis.

\section{References}

[1] A. Goetz, C. Ryll-Nardzewski, On bases of abstract algebras, 13ull. Acad. Pol. Sci., Sér. math., astr. et phys., 8 (1960), pp. 157-161.

[2] G. Grätzer, A theorem on doubly transitive permutation groups with application to universal algebras, Fund. Math. 53 (1963), pp. 25-41.

[3] E. Marczewski, Independence in some abstract algebras, Bull. Acad. Pol. Sci., Sér. math., astr. et phys., 7 (1959), pp. 611-616.

[4] - Independence and homomorphisms in abstract algebras, Fund. Math. 50 (1961), pp. 45-61.

[5] W. Narkiewicz, Independence in a certain class of abstract allgetris, ibidem 50 (1962), pp. 333-340.

[6] - A note on $v^{*}$-algebras, ibidem 52 (1963), pp. 289-290.

[7] K. Urbanik, A representation theorem for MTarezews7ii's algebras, ibilem 48 $\{1960\}$, pp. 147-167.

[8] - A representation theorem for $v^{*}$-algebras, ibidem 52 (1963), 1p]. 291-317.

MATHEMATICAL INSTITUTE, WROCEAW UNIVERSITY

MATHEMATICAL INSTITUTE OF THE POLISH ACADEMY OF SCIENCES

Reçu par la Rédaction le \%.12.1962

\section{Convergence functions and their related topologies*}

by

D. Kent (Albuquerque, N. Mex.)

\section{Introduction}

A convergence function is a correspondence between the filters on a given set $S$ and the subsets of $S$ which specifies which filters converge to which points of $S$. This concept is defined to include types of convergence which are more general than that defined by specifying a topology on $S$. Thus a convergence function may be regarded as a generalization of a topology.

Various generalizations of the latter concept have been made in the past with the help of convergence criteria; structures of this type have been identified with such names as "limitierung", "psendo-topologie", and "pretopologie". These latter structures may be regarded as special cases of convergence functions, more topology-like than the basic struetures which we investigate.

The method used here to study the convergence function is to place it in the ordered environment of a complete lattice $\mathcal{C}(S)$, whose elements are all the convergence functions on an arbitrary set $S$. Letting $q$ be an arbitrary convergence function on $S$, we associate with $q$ various topologies which are related to $q$ in a more or less natural way. To associate topologies with $q$ systematically, the concept of linkage function is introduced. A linkage function may be regarded as a method for obtaining a topology from a convergence function which is valid for any convergence function in $\mathcal{C}(\boldsymbol{S})$.

We investigate and compare four fundamental linkage functions. The first section introduces some relevant definitions, gives certain structural properties of $\mathcal{C}(S)$, and defines what is perhaps the simplest and most natural of linkage functions. A different linkage function is investigated in each of the remaining three sections.

* Research supporterl by the National Science Foundation, Grant NSF-G-21219. The work reported fortial fulfilment of the requirements for the Ph. D. Degree at the University of New Mexico. 